\title{
ESTUDIO MICROSCÓPICO DE MIEL Y POLEN APÍCOLA DE LA PROVINCIA DE SEVILLA
}

\author{
Pedro L. ORTIZ e Inmaculada FERNÁNDEZ
}

\begin{abstract}
RESUMEN. El objetivo del presente trabajo es poner de manifiesto cuál es la flora de mayor interés apícola en las áreas de sierra de la provincia de Sevilla. Se han estudiado al microscopio óptico veinte muestras de miel y dos de polen procedentes de diversas localidades de la provincia. Los resultados ponen de manifiesto que la principal fuente de miel para Apis mellifera L. es el néctar de las flores, si bien, a escala local, en Sierra Morena la mielada llega a ser muy valiosa como fuente de miel. Como principales fuentes de néctar cabe destacar a Echium plantagineum L., Eucalyptus camaldulensis Dehnh., Lavandula stoechas L., Thymbra capitata (L.) Cav. y Helianthus annuus L., mientras que de polen lo son Cistus albidus L., C. ladanifer L., Quercus spp., Myrtus communis L. y Papaver rhoeas L.
\end{abstract}

Palabras clave: Miel, polen, Melitopalinología, flora apícola, Sevilla.

ABSTARCT. Twenty honey and two pollen samples from different localities of the mountainous areas in the province of Seville (Spain) have been studied by light microscopy, in order to show the plants prefered by bees. The results point out that the nectar from flowers is the main honey source in the region, though locally honey-dew can be very valuable as raw material of honey. Echium plantagineum L., Eucalyptus camaldulensis Dehnh., Lavandula stoechas L., Thymbra capitata (L.) Cav. and Helianthus annuus L. are notable as supplying nectar to Apis mellifera L., while Cistus albidus L., C. ladanifer L., Quercus spp., Myrtus communis L. and Papaver rhoeas L. are interesting as pollen sources for honeybees.

Key words: Honey, pollen, Melitopalynology, honey-bee flora, Seville.

\section{INTRODUCCIÓN}

Los estudios melitopalinológicos, en su gran mayoría, tienen como finalidad dilucidar el origen botánico de las mieles. Sin embargo, sólo una parte del polen que se encuentra en el sedimento de la miel ha llegado a ella incluído en el néctar y por ello es indicio de su origen floral; el resto ha sido añadido a la miel posteriormente, bien por las abejas en el interior de la colmena, bien por el apicultor durante la extracción, y es indicativo de las fuentes de polen empleadas por esas abejas. El objetivo de este trabajo es también poner de manifiesto las preferencias alimenticias de Apis mellifera L. Para ello, los datos proporcionados por el análisis microscópico del sedimento polínico de las mieles y de cargas de polen han sido confrontados con otros referentes a la potencialidad nectarífera y polinífera de las especies en cuestión.

Con anterioridad a este trabajo se han llevado a cabo diversos estudios melitopalinológicos en Andalucía Occidental, entre ellos los de Pozo Lora (1970), Tello Porras (1982), Gómez Ferreras y Sáenz Lain (1985), Ortiz (1985, 1988, 1990 a y b) y Ortiz et al. (1990). 


\section{ÁREA DE ESTUDIO}

El área de estudio comprende las zonas de sierra de la provincia de Sevilla, que corresponden a la Sierra Norte y a las Sierras Subbéticas. La primera ocupa el tercio septentrional de la provincia, al $\mathrm{N}$ del Guadalquivir, y constituye una fracción del sistema orográfico de Sierra Morena. Las segundas son un grupo de pequeñas sierras que se alinean en el flanco SE de la provincia.

Las montañas de la Sierra Norte sevillana, de formas redondeadas, poco agrestes y de altitudes modestas, están formadas por materiales paleozoicos, principalmente graníticos, pizarrosos, calizos y cuarzíticos. Las montañas Subbéticas sevillanas, algo más elevadas y abruptas, están formadas por diversos materiales mesozoicos: margas arcillosas o yesosas, margocalizas y calizas metamórficas.

En la Sierra Norte el bosque potencial (encinar o alcornocal), a veces bien conservado, posee gran diversidad de especies. No obstante la vegetación boscosa está, en general, muy degradada y aparece sustituída por jarales en las zonas secas y brezales en las más húmedas. En muchos lugares el monte alto, con encinas o alcornoques y de forma local melojos o quejigos, se encuentra adehesado. Existen también amplias áreas dedicadas al cultivo de eucaliptos. En las Sierras Subbéticas las zonas no demasiado accidentadas están dedicadas a cultivos, principalmente de olivos, cereales y girasol. Donde los cultivos no son posibles, la vegetación está constituída por matorrales con especial abundancia de Labiadas.

\section{MATERIAL Y MÉTODOS}

Se ha llevado a cabo un estudio microscópico sobre veinte muestras de miel y dos de polen obtenidas directamente de los apicultores que las cosechan. Dichas muestras proceden de las siguientes localidades sevillanas: Los Corrales, El Saucejo, Morón de la Frontera, Montellano, El Madroño, El Ronquillo, Almadén de la Plata, El Real de la Jara, Castilblanco de los Arroyos, Cantillana, El Pedroso, Cazalla de la Sierra, Constantina y La Puebla de los Infantes. Las cuatro primeras corresponden a las Sierras Subbéticas y el resto a la Sierra Norte.

El análisis cuantitativo de las muestras de miel se ha efectuado sobre preparaciones microscópicas elaboradas sin ningún tratamiento químico, siguiendo básicamente el método descrito por Maurizio (1939, sec. Maurizio, 1979b) y contándose separadamente granos de polen y elementos indicadores de mielada.

El análisis cualitativo, en cambio, tanto de muestras de miel como de polen, se ha llevado a cabo sobre preparaciones microscópicas acetolizadas, elaboradas según el método de Erdtman (1960) ligeramente modificado. Siguiendo las indicaciones de Vergeron (1964) e I.C.B.B. of I.U.B.S. (1970, 1978) se han contado e identificado al menos 1200 granos de polen repartidos en cuatro preparaciones diferentes de cada muestra.

Los tipos polínicos se han identificado utilizando principalmente el atlas polínico de Valdés et al. (1987a), las preparaciones de referencia de la Palinoteca del Departamento de Biología Vegetal y Ecología de la Universidad de Sevilla y la clave palinológica de Moore y Webb (1978). Se ha tenido en cuenta, además, el área de distribución de cada especie (Valdés et al., 1987b) y la experiencia personal en el campo.

Siempre que ha sido posible se ha identificado el tipo polínico a nivel de especie. En caso contrario se ha llegado a nivel genérico o de tipo polínico que incluye 
géneros afines. Cuando es posible se añade, al nombre del género o tipo, una pequeña lista de las especies a las que se puede atribuir el polen en cuestión. Si dentro de esta lista alguna especie tiene mayor probabilidad de ser la representada en el espectro, ésta se subraya.

En el caso de las muestras de polen, a partir de los porcentajes obtenidos en el análisis cualitativo, referidos al número total de granos de polen por muestra, se han calculado otros porcentajes referidos al volumen total de cada muestra. Para ello, los porcentajes referidos al número de granos de polen de cada tipo polínico se multiplican por un factor de corrección proporcional al volumen de cada uno (Louveaux, 1958b). Las cifras resultantes de estos productos se transforman de nuevo en porcentajes respecto a la suma de todas esas cifras en cada muestra.

Una descripción detallada de los métodos empleados puede verse en Ortiz (1991).

\section{RESULTADOS}

En el Cuadro I se muestran los resultados del análisis cuantitativo de las muestras de miel. En general el sedimento de las muestras de miel estudiadas es abundante, predominando entre ellas las pertenecientes a las clases III y V de Maurizio (1949, sec. Maurizio, 1979b) y no incluyéndose ninguna en las clases I y IV. Con

\begin{tabular}{|c|c|c|c|c|}
\hline M.M. & N.G.P. & N.E.I.M. & N.E.B.T. & CLASE \\
\hline $\mathrm{Co}$ & 442.400 & 5.200 & 447.600 & III \\
\hline $\mathrm{Co}_{1}$ & 76.900 & 5.350 & 82.250 & II \\
\hline $\mathrm{Sa}^{2}$ & 28.166 & 1.966 & 30.132 & II \\
\hline MF & 4.945 .500 & 10.500 & 4.956 .000 & $\mathrm{~V}$ \\
\hline Mo & 1.121 .000 & 9.500 & 1.130 .500 & V \\
\hline $\mathrm{Ma}$ & 2.072 .000 & 117.700 & 2.189 .700 & V \\
\hline Ro & 2.538 .000 & 12.600 & 2.550 .600 & V \\
\hline $\mathrm{Ro}_{2}$ & 245.864 & 7.600 & 253.464 & III \\
\hline $\mathrm{Ro}_{3}$ & 118.200 & 21.066 & 139.266 & III \\
\hline $\mathrm{Ro}_{4}^{3}$ & 118.650 & 20.250 & 138.900 & III \\
\hline $\mathrm{AP}^{4}$ & 129.000 & 19.800 & 148.800 & III \\
\hline RJ & 5.544 .000 & 19.500 & 5.563 .500 & $\mathrm{~V}$ \\
\hline $\mathrm{CA}$ & 375.300 & 5.400 & 380.700 & III \\
\hline $\mathrm{Cn}$ & 141.000 & 500 & 141.500 & III \\
\hline $\mathrm{Pe}_{1}$ & 60.200 & 13.500 & 73.700 & II \\
\hline $\mathrm{Pe}_{2}$ & 80.400 & 5.666 & 86.066 & II \\
\hline $\mathrm{CS}^{2}$ & 2.650 .000 & 55.400 & 2.705 .400 & V \\
\hline $\mathrm{Ct}$ & 5.476 .000 & - & 5.476 .000 & V \\
\hline PI & 1.854 .330 & 27.660 & 1.881 .990 & V \\
\hline $\mathrm{PI}_{2}^{1}$ & 487.332 & 4.532 & 491.864 & III \\
\hline
\end{tabular}

Cuadro I.- Resultados del análisis cuantitativo de las muestras de miel (M.M.). Los números de granos de polen (N.G.P.), de elementos indicadores de mielada (N.E.I.M.) y de elementos botánicos totales (N.E.B.T.) se refieren a 10 gramos de miel. Las clases corresponden a las establecidas por Maurizio (1949, sec. Maurizio, 1979b). Co, Los Corrales; Sa, El Saucejo; MF, Morón de la Frontera; Mo, Montellano; Ma, El Madroño; Ro, El Ronquillo; AP, Almadén de la Plata; RJ, El Real de la Jara; CA, Castilblanco de los Arroyos; Cn, Cantillana; Pe, El Pedroso; CS, Cazalla de la Sierra; Ct, Constantina; PI, La Puebla de los Infantes. 
excepción de la muestra de Constantina en que no se encontró ningún elemento indicador de mielada, el sedimento está compuesto en todos los casos tanto por granos de polen como por elementos indicadores de mielada (hifas y esporas de hongos). En las muestras de las Sierras Subbéticas los elementos indicadores de mielada son escasos; en cambio, en buena parte de las mieles de la Sierra Norte el número de estos elementos es considerable e incluso elevado en dos de ellas (El Madroño y Cazalla de la Sierra).

En el Cuadro II se presentan resumidamente los resultados obtenidos en el análisis cualitativo. Los tipos polínicos presentes únicamente en una o dos muestras con porcentajes de 1 ó 2 han sido omitidos como tales en este cuadro, habiéndose agrupado con tipos de la misma familia en unos casos, o incluyéndose en el apartado «Otros tipos» en otros.

En el análisis cualitativo de las muestras de miel y polen se han encontrado 68 tipos polínicos presentes en alguna de las muestras al menos en un 1\%,34 de los cuales están representados en las muestras de miel de las Sierras Subbéticas, 54 en las de la Sierra Norte y 12 en las de polen. El número de estos tipos por muestra oscila entre 9 y $20(\bar{x} \pm$ E.S. $=15,8 \pm 1,9 ; n=5)$ para las muestras de miel de las Sierras Subbéticas y entre 7 y $19(\bar{x} \pm$ E.S. $=12,9 \pm 0,9 ; n=15)$ para las muestras de miel de la Sierra Norte. En las muestras de polen estudiadas este número es 3 en una y 10 en otra.

Estos tipos polínicos pertenecen a 32 familias, siendo Asteraceae, Fabaceae, Cistaceae y Lamiaceae, por este orden, las que contribuyen con un mayor número de tipos, mientras que 20 de ellas sólo están representadas por un tipo.

En la Figura 1 se resaltan los resultados más destacables del análisis cualitativo. El polen de Echium plantagineum L., Cistus spp. (C. albidus L. y/o C. crispus L.) y Eucalyptus spp. está representado en gran número de muestras de toda la provincia. El de Helianthus annuus L., Ononis spp., Thymbra capitata (L.) Cav. y Papaver rhoeas L. está presente en todas o casi todas las muestras de las Sierras Subbéticas. El de Papaver rhoeas, además, es dominante en una de las mieles de Sierra Morena. En las muestras de Sierra Morena también está bien representadoel de Cistus spp. (C. ladanifer L. y/o C. populifolius L.), Quercus spp., Lavandula stoechas L. y Myrtus communis L. Finalmente, del polen de tipo Raphanus raphanistrum, Erica arborea L., Erica spp. (E. australis L. y/o E. umbellata L.), tipo Cytisus scoparius, Castanea sativa Miller, Cytinus hypocistis (L.) L. y Rubus ulmifolius Schott cabe destacar su presencia con porcentaje considerable en alguna de las muestras de Sierra Morena.

\section{DISCUSIÓN}

Nuestros resultados muestran que aunque la mielada haya sido parte de la materia prima de las mieles de las Sierras Subbéticas no tiene demasiada importancia como fuente de miel en esta comarca. Se evidencia, por el contrario, que la mielada es bastante valiosa como fuente de miel en la Sierra Norte, siendo incluso muy sobresaliente a escala local. Ortega (1986) cita a la mielada de Quercus rotundifolia Lam. como una fuente de miel muy importante en esta comarca, y en Sierra Morena en general, hasta el punto que motiva trashumancia entre los meses de Agosto y Octubre. Otras especies presentes en la Sierra Norte sevillana y que han sido citadas como fuentes de mielada son Quercus spp. (Ortega, l.c.), Populus alba L., P. nigra L. y Castanea sativa (Crane y Walker, 1985) y Salix atrocinerea Brot. (Ortiz, 1991).

Helianthus annuus (girasol) es una planta visitada por las abejas para recoger su néctar principalmente, siendo su polen poco recolectado habitualmente (Fonta et 
al., 1985; Fell, 1986). Varios autores han observado abejas recolectoras de néctar de girasol, con el cuerpo cubierto de polen de esta especie, sin hacer ningún intento de empaquetarlo en sus corbículas e incluso desprendiéndose activamente de él (Synge, 1947; Louveaux, 1959; Parker, 1981). En contraste con estas observaciones, Crane et al. (1984) citan a Helianthus annuus como fuente de polen de primera magnitud en algunos sitios, si bien la destacan como recurso nectarífero de primer orden en un mayor número de lugares. El polen de Helianthus annuus se encuentra infrarrepresentado en los espectros polínicos de las mieles, ya que mieles monoflorales experimentales de girasol contienen menos de 3000 granos de polen en 10 gramos (Demianowicz, 1964). Las muestras de miel en que hemos encontrado polen de Helianthus annuus (vease Cuadro II), sin ser monoflorales de esta especie, presentan contenidos absolutos en granos de polen de la misma muy superiores al encontrado por Demianowicz (1.c.). Esto indica que estas abejas han recolectado cargas de polen de esta especie y a partir de éstas se han contaminado las mieles. Sin duda Helianthus annuus ha sido una importantisima fuente de néctar para las abejas productoras de estas mieles, a la vez que han recogido su polen de manera destacable. Nuestros resultados muestran que el girasol tiene un enorme interés apícola en las Subbéticas y es interesante puntualmente en Sierra Morena.

Echium plantagineum es una especie de la que Apis mellifera obtiene tanto néctar como polen (Corbet y Delfosse, 1984), tendiendo este último a aparecer hiperrepresentado en los espectros polínicos de las mieles (Espada, 1984). De nuestros resultados se deduce que esta especie no tiene demasiado interés apícola en el sur de la provincia, pero en el norte es una importante fuente de miel y polen.

Algunas Brassicaceae son interesantes como fuente de polen en la Sierra Norte, al menos localmente. Puesto que muchas Brassicaceae son productoras de néctar (Crane, 1979; Maurizio, 1979a; Rita, 1983; Crane et al., 1984; Soler et al., 1986) es probable que en alguna medida también sean recursos nectaríferos usados por Apis mellifera.

Aunque las especies del género Cistus presentes en el territorio estudiado producen néctar, lo más destacable de las mismas es su potencialidad polinífera (Talavera et al., 1988). Nosotros hemos observado que Apis mellifera, cuando visita estas especies, se interesa por ambas recompensas. Por todo ello, y a la vista de los resultados obtenidos, podemos decir que Cistus albidus en las Sierras Subbéticas y Cistus crispus y $C$. ladanifer, principalmente, en la Sierra Norte son importantes recursos polínicos para las abejas de ambas comarcas, a la vez que suponen un aporte nectarífero que localmente podría tener cierto interés. Tenemos constancia de que en primavera, coincidiendo con la floración de estas especies, un gran número de colmenas, procedentes de Levante sobre todo, son trasladadas a los extensos jarales de la Sierra Norte sevillana para aprovechar la inmensa producción de polen de los mismos.

En la mitad occidental de la Sierra Norte, al menos localmente, Erica arborea y, en menor medida, E. australis y E. umbellata son explotadas de manera notable por las abejas domésticas. Dado el elevadísimo contenido polínico de las mieles en que está presente el polen de Erica spp., no cabe duda de que estas especies han sido empleadas como recursos poliníferos importantes, aunque lógicamente Apis mellifera también habrá explotado el néctar que estas especies producen (Crane et al., 1984; Herrera, 1985).

Casi todas las especies de nuestro territorio pertenecientes a la tribu Genisteae presentan granos de polen que se pueden incluir en el tipo Cytisus scoparius (Valdés et al., 1987a). La mayoría de las especies de esta tribu ofrecen polen como única recompensa a los polinizadores (Herrera, 1985; Talavera et al., 1988). Nuestros 


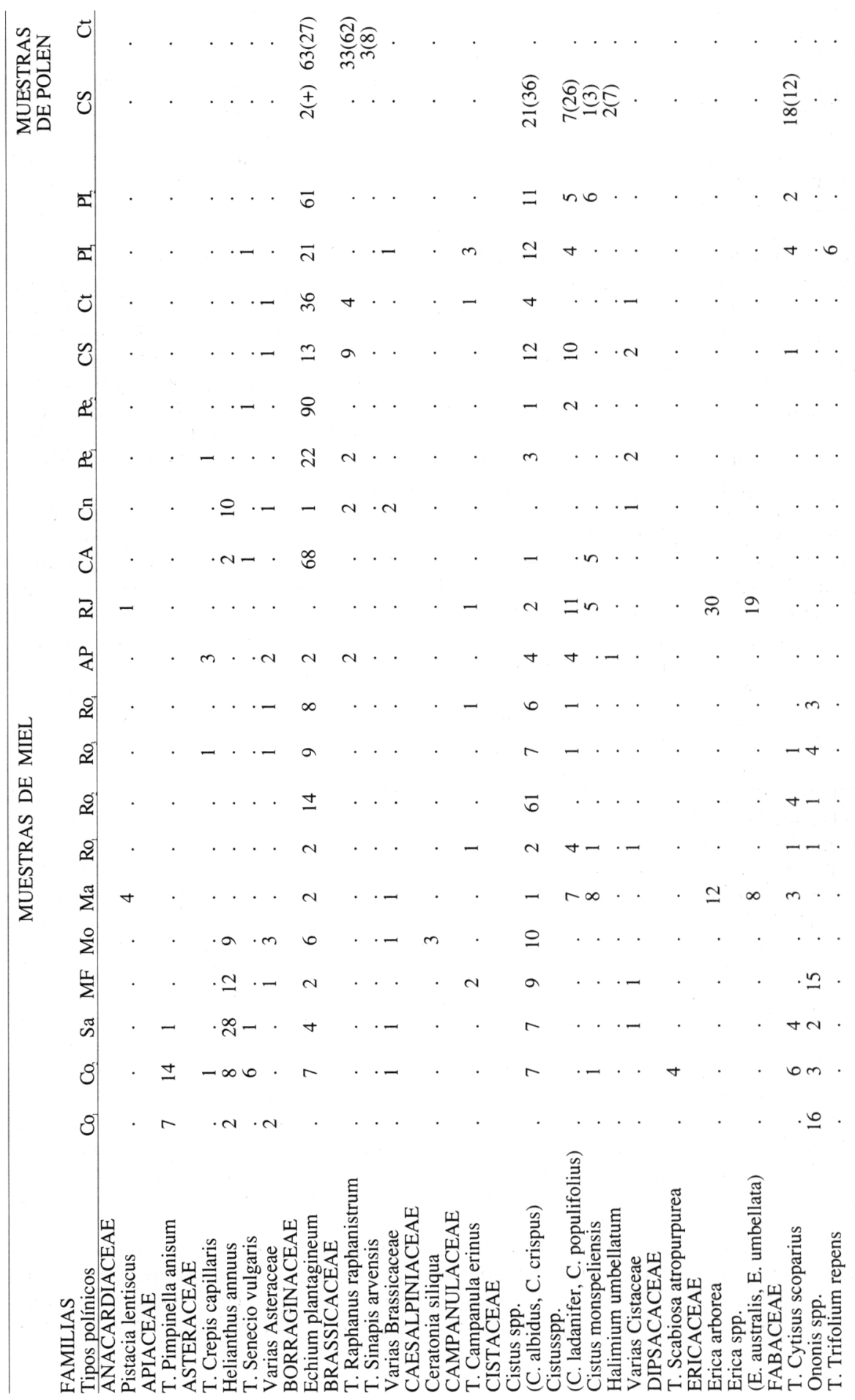









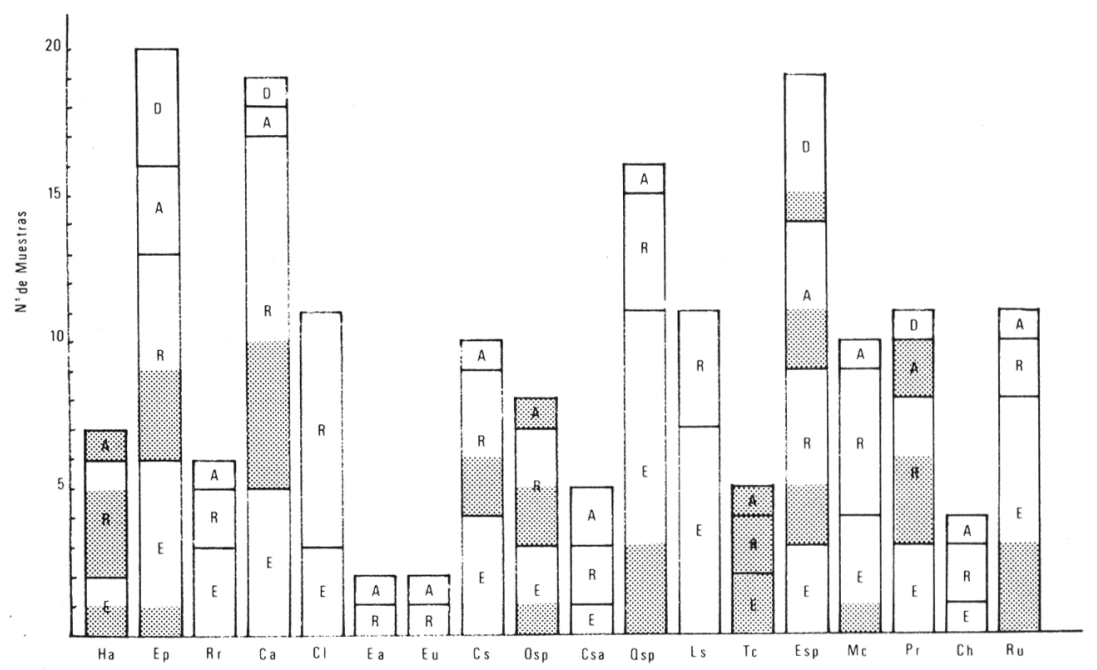

Figura 1.- Las barras indican el número de muestras de miel y polen en que aparecen, incluídos en cada clase de frecuencia, los tipos polínicos mejor representados. Dentro de cada barra las áreas en blanco corresponden a muestras de Sierra Morena y las sombreadas a muestras de las Sierras Subbéticas. D: Dominante (>45\%); A: Acompañante (16-45\%); R: Raro (3-15\%); E: Esporádico $(<3 \%)$. Ha: Helianthus annuus; Ep: Echium plantagineum; Rr: tipo Raphanus raphanistrum; Ca: Cistus spp. (C. albidus y/o C. crispus); Cl: Cistus spp. (C. ladanifer y/o C. populifolius); Ea: Erica arborea; Eu: Erica spp. (E.australis y/o E. umbellata); Cs: tipo Cytisus scoparius; Osp: Ononis spp.; Csa: Castanea sativa; Qsp: Quercus spp.; Ls: Lavandula stoechas; Tc: Thymbra capitata; Esp: Eucalyptus spp.; Mc: Myrtus communis; Pr: Papaver rhoeas; Ch: Cytinus hypocistis; Ru: Rubus ulmifolius.

resultados apuntan a que alguna especie con polen de este tipo tiene interés apícola en la Sierra Norte, al menos localmente y como fuente de polen. De las especies incluídas en dicho tipo polínico, la más abundante en este área es Retama sphaerocarpa (L.) Boiss., una de las pocas especies de Genisteae que produce néctar (Herrera, l.c.; Talavera et al., l.c.), por lo que la presencia de este tipo polínico en algunas de las mieles estudiadas puede responder parcialmente a su uso como recurso nectarífero.

Algunas especies de Ononis no producen néctar (Herrera, 1988; Ortiz, 1991) y otras producen cantidades muy pequeñas por flor (Talavera et al., 1988). Dada la relativa y extrema abundancia del sedimento de las mieles $\mathrm{Co}_{1}$ y $\mathrm{MF}$, respectivamente, podemos afirmar que el polen de Ononis es recogido de modo importante por las abejas en las Sierras Subbéticas. Es posible que alguna de las especies a las que puede pertenecer el polen de estas mieles sea productora de néctar, y que éste sea recogido por Apis mellifera. Si ocurriera así, no es probable que ésta fuese una fuente importante de néctar, ya que no se ha encontrado ninguna referencia bibliográfica que señale alguna especie de Ononis como de importancia melífera.

Castanea sativa es una especie productora de néctar, si bien lo mas destacable es su potencialidad polinífera (Talavera et al., 1988). Su polen es de tamaño pequeño y aparece fuertemente hiperrepresentado en las mieles (I.C.B.B. of I.U.B.S., 1978; Crane et al., 1984). Nuestros resultados muestran que Castanea sativa es interesante como recurso nectarífero y polinífero en aquella parte de la provincia donde está presente: el cuadrante nororiental. 
Puesto que las especies del género Quercus tienen polinización anemófila y no producen néctar, la presencia de su polen en la miel se debe a la contaminación de ésta a partir del recolectado por las abejas, ya sea producida esta contaminación por las propias abejas en el interior de la colmena o bien por el apicultor durante el proceso de extracción (Louveaux, 1958a; Maurizio, 1979b). Por tanto de los resultados obtenidos se deduce que estas plantas son fuentes de polen destacables para las abejas en la Sierra Norte de Sevilla. Por su abundancia en la zona destacan Quercus rotundifolia Lam., $Q$. coccifera L. y en menor medida $Q$. suber L.

Son diversos los autores que señalan el caracter nectarífero de Lavandula stoechas (Muñoz y Devesa, 1987, p.e.) y los que la reconocen como planta melífera importante (Crane et al., 1984, p.e.). El polen de esta especie aparece infrarrepresentado en los espectros polínicos de las mieles (Stanley y Linskens, 1974), por lo que de nuestros resultados se desprende que Lavandula stoechas es destacable como fuente de néctar para Apis mellifera en la Sierra Norte. Asimismo, su polen también es explotado por las abejas.

Talavera et al. (1988) señalan que, en las Subbéticas sevillanas, Thymbra capitata, por su potencial nectarífero y su abundancia, puede ser explotada apícolamente para la obtención de miel monofloral. A ello contribuiría su floración estival (Arroyo, 1988), cuando las colonias de abejas están preparadas para almacenar reservas de miel. Al igual que el de Lavandula stoechas y el de Lamiaceae, en general, el polen de esta especie también aparece infrarrepresentado en los espectros de las mieles (Serra et al., 1986). Como ya hemos comentado en el caso del girasol, algunas de las mieles de las Subbéticas, sin ser monoflorales de Thymbra capitata, presentan un número absoluto de granos de polen de esta especie demasiado elevado para un tipo polínico con tendencia a aparecer infrarrepresentado. Concluimos pues, que Thymbra capitata es un recurso nectarífero de primera magnitud en las Sierras Subbéticas y que su polen también es recolectado por Apis mellifera.

El polen de Eucalyptus encontrado en las muestras estudiadas pertenece, en exclusiva en las de las Subbéticas y mayoritariamente en las de Sierra Morena, a Eucalyptus camaldulensis Dehnh. Esta especie es una buena productora tanto de miel como de polen (Crane et al., 1984) y su polen aparece suprarrepresentado en los espectros polínicos de las mieles (Espada, 1984). De nuestros resultados se desprende que el néctar de Eucalyptus camaldulensis es parte importante de la materia prima de las mieles que se producen en Sevilla, y que su polen es un aporte considerable a la dieta de las colonias de abejas que han producido estas mieles.

Myrtus communis es una planta entomófila que ofrece polen como única recompensa a los polinizadores (Talavera et al., 1988). Lo mismo ocurre con Papaver rhoeas (Weber El- Ghobary, 1984). Myrtus communis es destacable como recurso polínico en la Sierra Norte y Papaver rhoeas forma parte importante de la dieta polínica de la abeja doméstica en las Sierras Subbéticas y, localmente, en Sierra Morena.

Finalmente, Cytinus hypocistis y Rubus ulmifolius son también plantas entomófilas que a diferencia de las dos anteriores ofrecen néctar y polen como recompensas (Ortiz, 1991). Ambas son localmente importantes como fuente de alimento para la abeja melífera en la Sierra Norte de Sevilla.

\section{CONCLUSIONES}

Es destacable que los jarales de la Sierra Norte sevillana ofrecen una enorme cantidad de polen en primavera, cuando la demanda y, por tanto, la recogida del mismo en la colmena alcanzan sus niveles más elevados. Probablemente la producción 
polínica de los jarales sea el elemento de mayor interés apícola en la Sierra Norte de Sevilla. En la misma zona, es también de gran interés el flujo nectarífero de Eucalyptus camaldulensis durante los meses estivales, momento en que las colmenas están preparadas normalmente para almacenar grandes cantidades de miel.

En las Sierras Subbéticas los elementos más destacables desde el punto de vista apícola son los aportes nectaríferos èstivales de Thymbra capitata y Helianthus annuus. Sin duda esta última especie tiene aún mayor importancia en la vecina Campiña.

AGRADECIMIENTOS. Deseamos expresar nuestro agradecimiento a D. F. J. García Ugidos por facilitarnos parte del material estudiado y al Prof. S. Talavera y a la Dra. M. J. Díez por su ayuda y consejos. El presente trabajo ha sido financiado con cargo a los proyectos de la CAICYT 264/82 y PA 85-297.

\section{BIBLIOGRAFIA}

ARROYO, J. -1988- Fenología de la floración en especies del matorral del Sur de España. Lagascalia 15 (Extra): 593-606.

CORBET, S.A. \& E.S. DELFOSSE -1984- Honeybees and the nectar of Echium plantagineum L. in south-eastern Australia. Austral. J. Ecol. 9: 125-139.

CRANE, E. -1979- The flowers honey comes from, in E. CRANE (ed.) Honey. A comprehensive survey. Heinemann. London: 115-153.

CRANE, E. \& P. WALKER -1985-Important honeydew sources and their honeys. Bee World 66 (3): 105-112.

CRANE, E., P. WALKER \& R. DAY -1984-Directory of important world honey sources. Int. Bee Res. Assoc. London.

DEMIANOWICZ, Z. -1964- Charakteristik der einartenhonige. Ann. Abeille 7 (4): 273-288.

ERDTMAN, G. -1960- The acetolysis method. A revised description. Svensk. Bot. Tidskr. 54 (4): 561-564.

ESPADA, T. -1984- Contribución al conocimiento de las mieles de producción nacional: espectro polínico de la miel de Brezo de Cataluña. Vida Apícola 11: 17-20.

FELL, R.D. -1986- Foraging behaviors of Apis mellifera L. and Bombus spp. on oilseed sunflower (Helianthus annuus). J. Kansas Entomol. Soc. 59 (1): 72-81.

FONTA, C., M.H. PHAM-DELEGUE, R. MARILLEAU \& C. MASSON -1985- Rôle des nectars de tournesol dans le comportement des insectes pollinisateurs et analyse qualitative et quantitative des éléments glucidiques de ces sécrétions. Acta Oecol./Oecol.Appl.6 (2): $175-186$.

GOMEZ FERRERAS, C. y C. SAENZ LAIN -1985- Estudio del sedimento polínico de las mieles de la Reserva Biológica de Doñana (Huelva, España). Anales Asoc. Palinol. Lengua Esp. 2: 369-374.

HERRERA, J. -1985- Nectar secretion patterns in southern Spanish mediterranean shrublands. Israel J. Bot. 34: 47-58.

HERRERA, J. -1988- Datos sobre biología floral en la flora de Andalucía Oriental. Lagascalia 15 (Extra): 607-614.

I.C.B.B. of I.U.B.S. -1970- Methods of Melissopalynology. Bee World 51 (3): 125-138.

I.C.B.B. of I.U.B.S. -1978- Methods of Melissopalynology. Bee World 59 (4): 139-157.

LOUVEAUX, J. - 1958a- Recherches sur l'origine dans le miel du pollen de plants entomophiles dépourvues de nectaires. Ann. Abeille 1 (2): 89-92.

LOUVEAUX, J. -1958b- Recherches sur la récolte du pollen par les abeilles (Apis mellifera L.). Ann. Abeille 1 (3): 113-188.

LOUVEAUX, J. -1959- Recherches sur la récolte du pollen par les abeilles (Apis mellifera L.) (fin). Ann. Abeille 2 (1): 13-111.

MAURIZIO, A. - 1979a- How bees make honey, in E. CRANE (ed.) Honey. A comprehensive survey. Heinemann. London: 77-105. 
MAURIZIO, A. -1979b- Microscopy of honey, in E. CRANE (ed.) Honey. A comprehensive survey. Heinemann. London: 240-257.

MOORE, P.D. \& J.A. WEBB -1978- An illustrated guide to pollen analysis. Hodder \& Stoughton. London.

MUNOZZ, A. y J.A. DEVESA -1987-Contribución al conocimiento de la biología floral del género Lavandula L. II. Lavandula stoechas L. subsp. stoechas. Anales Jard. Bot. Madrid 44 (1): 63-78.

ORTEGA, J.L. -1986- Flora de interés apícola de la España Peninsular. Actas II Congr. Nac. Apic. (Gijón, 1984): 156-171.

ORTIZ, P.L. -1985- Análisis polínico de mieles y celdillas de las sierras del sur de Córdoba (España). Anales Asoc. Palinol. Lengua Esp. 2: 353-360.

ORTIZ, P.L. -1988-Estudio melitopalinológico en El Andévalo (Huelva). Anales Asoc. Palinol. Lengua Esp. 4: 64-72.

ORTIZ, P.L. -1990a- Aportación melitopalinológica al conocimiento de la flora apícola del norte de Córdoba. Lagascalia 15 (2): 165-177.

ORTIZ, P.L. -1990b-Contribución al conocimiento de la flora apícola gaditana. Lagascalia $16(2): 199-210$.

ORTIZ, P.L. -1991- Melitopalinología en Andalucía Occidental. Tesis Doctoral. Universidad de Sevilla.

ORTIZ, P.L., I. FERNANDEZ y M. MARTIN CACAO -1990-Estudio melitopalinológico en la comarca de Aracena (Huelva). Lagascalia 16 (1): 61-76.

PARKER, F.D. -1981- How efficient are bees in pollinating sunflowers? J. Kansas Entomol. Soc. $54(1): 61-67$.

POZO LORA, R. -1970- Investigaciones sobre mieles españolas. I: Espectro polínico de la miel de San Calix to (Hornachuelos, Córdoba, España). Arch. Zootecnia 19 (76): 361-374.

RITA, J. -1983-Flora melífera de la provincia de Lérida. Excma. Diputación de Lérida. Lérida.

SERRA, J., A. GOMEZ PAJUELO y J. GONELL GALINDO -1986- Estudio del espectro polínico de la miel de espliego (Lavandula latifolia Mod. con Lavandula pedunculata Cav.) producida en Cuenca, Guadalajara y Soria. Actas II Congr. Nac. Apic. (Gijón, I984): $137-$ 144.

SOLER, L., T. ESPADA y A. GOMEZ PAJUELO -1986- Estudio de la evolución estacional de la flora melífera y polinífera de Caldes de Montbui (Barcelona) mediante melisopalinología. Actas II Congr. Nac. Apic. (Gijón, 1984): 60-69.

STANLEY, R.G. \& H.F. LINSKENS -1974-Pollen. Biology Biochemistry Management. Springer-Verlag. Berlin, Heidelberg, New York.

SYNGE, A.D. -1947- Pollen collection by honeybees (Apis mellifera). J. Anim. Ecol. 16: 122 138.

TALAVERA, S., J. HERRERA, J. ARROYO, P.L. ORTIZ y J.A. DEVESA -1988- Estudio de la flora apícola de Andalucía Occidental. Lagascalia 15 (Extra): 567-591.

TELLO PORRAS, E. -1982- La miel de Aracena. Arch. Zootecnia 31 (121): 293-303.

VALDES, B., M.J. DIEZ e I. FERNANDEZ (Eds.) -1987a-Atlas polínico de Andalucía Occidental. Inst. Des. Regional y Excma. Diputación de Cádiz. Sevilla.

VALDES, B., S. TALAVERA y E. FERNANDEZ GALIANO (Eds.) -1987b- Flora vascular de Andalucía Occidental. 3 vols. Ketres. Barcelona.

VERGERON, P. - 1964- Interprétation statistique des résultats en matière d'analyse pollinique des miels. Ann. Abeille 7 (4): 349-364.

WEBER EL-GHOBARY, M.O. -1984-Observation of flowering, pollen, nectar and pollen loads, due to Apis mellifera in some mediterranean plants. Les Colloques de l'INRA 21: 245-250.

(Aceptado para su publicación en Octubre de 1991)

Dirección de los autores: Departamento de Biología Vegetal y Ecología. Facultad de Biología. Apdo. 1095. 41080 Sevilla. 